Original article

\title{
The mediating role of borderline personality features in the relationship between childhood trauma and psychotic-like experiences in a sample of help-seeking non-psychotic adolescents and young adults
}

\author{
Mary Sengutta ${ }^{a, *}$, Łukasz Gawęda ${ }^{a, b}$, Steffen Moritz ${ }^{a}$, Anne Karow ${ }^{a}$ \\ a Department of Psychiatry and Psychotherapy, University Medical Center Hamburg Eppendorf, Hamburg, Germany \\ b II Department of Psychiatry, Medical University of Warsaw, Poland
}

\section{A R T I C L E I N F O}

\section{Article history:}

Received 24 July 2018

Received in revised form 27 October 2018

Accepted 26 November 2018

Available online 31 December 2018

\section{Keywords:}

Psychotic-like experiences

Childhood trauma

Borderline personality disorder

Borderline personality features

Anxiety

Depression

\begin{abstract}
A B S T R A C T
Objective: Psychotic-like experiences (PLEs) often occur across different non-psychotic disorders in adolescent and young adult population and are related to early trauma. However, the mechanisms of how exposure to early trauma shapes the risk of PLEs are unclear. In our study, we investigated whether borderline personality features and further non-psychotic symptoms, i.e. factors related to both PLEs and childhood trauma, may mediate the relationship between childhood trauma and PLEs.

Methods: Two hundred inpatients aged 16-21 years who were treated due to non-psychotic disorders were included. PLEs were assessed with the Prodromal Questionnaire (PQ-16). Childhood Trauma was assessed with the Adverse Childhood Experience Questionnaire (ACE). Borderline personality features were assessed by using the Borderline-Symptom Checklist (BSL-23). Presence and frequency of depressive symptoms and anxiety were assessed by Patient Health Questionnaire (PHQ-9) and Generalized Anxiety Disorder Questionnaire (GAD-7).

Results: A significant relationship between childhood trauma (ACE total score) and PLEs was found ( $\beta=0.30,95 \%$ CI 0.247-0.659). In particular, emotional neglect $(r=0.298, p<0.001)$ and sexual abuse $(r=0.264, p<0.001)$ were significantly associated with PLEs. Borderline personality features fully mediated the relationship of childhood trauma and PLEs ( $\beta=0.12,95 \% \mathrm{CI}:-0.019-0.370)$. Anxiety and Depression showed a significant, but partial mediation of the relationship.

Conclusion: Borderline personality features seem to be an important mediator of the relationship between childhood trauma and PLEs in adolescent patients with different non-psychotic psychiatric disorders. Theoretical and clinical implications are discussed.
\end{abstract}

(C) 2018 Elsevier Masson SAS. All rights reserved.

\section{Introduction}

Psychotic-like experiences (PLEs) can be defined as subclinical psychotic phenomena like perceptual anomalies and delusion-like experiences in the absence of manifest psychotic illness [1-3]. PLEs are common and mostly transient in the general population, with the highest prevalence being observed among children and adolescents [4-7]. Although PLEs often occurs in children and adolescents without impairments or pathological significance, if persisting over time these symptoms may become a source of functional impairment, an increased risk for a wide range of

\footnotetext{
* Corresponding author.

E-mail address: m.sengutta@uke.de (M. Sengutta).
}

nonpsychotic psychopathology, tendency to self-injurious behavior, and higher stress [6,8-12].

Approximately $80 \%$ of the 13 to 16 -year-old non-help seeking adolescents from community reported PLEs had a non-psychotic Axis-I disorder [12]. The presence of PLEs in community samples was associated with an increased severity of non-psychotic symptoms characterized by multiple co-occurring diagnoses [12], a significantly lower psychosocial function level and a poorer course of the illness $[3,13]$. This also holds for clinical samples. A study conducted on 108 adolescent outpatients (12-16 years) showed that $78 \%$ of those who reported PLEs also met the criteria for two or more non-psychotic psychiatric disorders [11] and the presence of PLEs were associated with poorer social and role functioning and increased severity of non-psychotic symptoms [14-16]. The occurrence of PLEs in adolescents seems closely linked to various psychiatric symptoms $[12,13,17-19]$. For example, 
the lifetime diagnosis of a major depressive or an anxiety disorder was associated with a significantly higher prevalence of PLEs [17].

In particular, Borderline Personality Disorder (BPD) or BPD features seem to be a risk factor for the development of PLEs; recent studies showed that up to $50 \%$ of the patients with BPD reported psychotic experiences, especially if they were exposed to childhood trauma [20-26]. In a recent study [27], more than a third of patients with BPD presented a variety of PLEs that particularly included alterations of perception. In the early days the term borderline reflected the idea that patients with borderline specific pathology were on the border to psychosis [28]. It was observed that patients with BPD tend to develop psychotic symptoms which would assume as the expression of a pathognomonic feature of the disorder [29]. Within psychodynamic perspective reality testing in patients with BPD is usually unimpaired, but may lose this ability under the influence of severe stress (particularly interpersonal difficulties) [30].

An important factor that may contribute both to the development of PLEs and BPD features is childhood exposure to traumatic life events. Indeed, studies consistently show a link between childhood trauma such as physical, sexual and emotional abuse and neglect and the development of psychotic-like experiences [31-33]. Adolescents who presented PLEs were more frequently exposed to physical abuse, neglect and domestic violence in their childhood or experienced discrimination such as bullying [34-36]. Similarly, the development of BPD is strongly related to childhood trauma [37] and it was suggested that childhood trauma could constitute an etiological factor for the development of BPD $[25,38]$. However, the interplay between exposure to childhood trauma, BPD features, anxiety, depression, and PLEs among adolescents and young adults has not yet been investigated.

The primary aim of the study was to fill this gap in our knowledge on the mechanisms related to personality features of the relationship between childhood trauma and PLEs in nonpsychotic adolescents and young adults referring to psychiatric service. Considering the findings that childhood trauma is putatively involved in the pathogenesis of both BPD and PLEs, we hypothesized that the presence of borderline personality features in adolescent patients with various non-psychotic psychiatric disorders serve as a mediator of the relationship between childhood trauma and PLEs in a sample of non-psychotic adolescent help-seekers. As mentioned above, individuals with depression and anxiety also often report PLEs [17]. Moreover, some studies emphasized the particular role of anxiety and depression regarding the relationship of traumatic experiences and PLEs $[35,39]$. Hence, based on the diagnostic heterogeneity of our sample and the existence of indices that symptoms of depression and anxiety could have an influence on the relationship between childhood trauma and PLEs as well, we performed additional analysis to prove their influence.

\section{Method}

\subsection{Participants}

The study cohort was obtained from 200 inpatients (133 women and 67 men) with a mean age of 18.7 years $(S D=1.85)$ who presented to the adolescent ward at University Medical Centre Hamburg Eppendorf, Department for Psychiatry and Psychotherapy (Germany) due to non-psychotic psychiatric disorders. All consecutive new inpatients were included in the study during 2015 and 2017. Patients with psychotic disorders were excluded from this study. A history of drug use or one or more comorbid disorders were not an exclusion criterion.

It was part of the general diagnostic approach of the ward to examine patients while enrollment with different diagnostic interviews and self-reporting questionnaires to verify their diagnosis and it will be during the treatment which takes mostly 8-10 weeks carefully proved till discharge. Ethical approval for the study was received from local ethics commission.

\subsection{Measures}

\subsubsection{Diagnostic instruments}

To assess current and lifetime Axis-I diagnosis and exclude patients with psychotic disorders all patients were routinely administered the Structured Clinical Interview for the Diagnostic and statistical manual of mental disorders, $4^{\text {th }}$ edition (DSM-IV) [40] at the point of beginning their inpatient treatment. For the assessment of personality disorders, we used the Structured Clinical Interview for DSM-IV Axis II Personality Disorders (SCID-II) [41].

\subsubsection{Clinical measures/Questionnaires}

Psychotic-like experiences were assessed using the Prodromal Questionnaire 16-item Scale (PQ-16) [42], which is the short version of 92-item Prodromal Questionnaire [43] to screen for the presence of PLEs and associated distress. The German translation was conducted by two clinical psychologists both fluent in English. A back-translation was performed by a native English-speaking translator. The self-rating questionnaire captures perceptual abnormalities (9 items), unusual thought content and paranoia (5 items) and negative symptoms ( 2 items). Each item can be marked true/false. For every item marked with true, patients have to rate the distress scale ranging from 0 (no distress) to 3 (severe). Scoring can be produced by summing all positive screened items (range $0-16$ ) as well as by a sum of the distress score (range 0-48). Cronbach's alpha for the PQ-16 is 0.77 .

Traumatic life events were assessed by using the Adverse Childhood Experience Questionnaire (ACE) [44], a 10 item questionnaire developed for the assessment of adverse life events in childhood and youth until the age of 18 years. Every item can be answered yes/no and can be summed to a general score which shows the quantity of burden. The items captured emotional, physical and sexual abuse as soon as emotional and physical neglect, separation from a parent, violence against the mother, substance abuse or dependence, mental illness and/or imprisonment of a member of the household. ACE has satisfactory psychometric characteristics, with Cronbach's alpha 0.75.

Borderline personality features were assessed by using the Borderline Symptom List (BSL-23) [45], a short version of the origin 95-item questionnaire [46] for the assessment of typical borderline symptomatology. This 23 item self-rating instrument contains those items from the long version that discriminate well between BPD and other disorders and had shown high levels of sensitivity. The items are quantitatively assessed on a 5-point Likert scale that ranges from 0 (not at all) to 4 (very strong) and assessed severity of Borderline personality disorder as well as sensitivity to change. Cronbach's alpha for the BSL-23 is 0.94 .

Depressive and Anxiety Symptoms were assessed by submodules of the Patient Health Questionnaire, German version (PHQ-D) [47], a screening tool for the assessment of common mental disorders. The PHQ-9 [48] is a nine-item questionnaire used for the screening of presence and frequency of depressive symptoms. Its items are based on the DSM-IV criteria for depression and figure depressive symptoms over the past two weeks including mood symptoms (e.g. sadness or irritability, anhedonia, loss of interests, guilt feelings and worthlessness), cognitive symptoms (concentration disorders, impaired decision-making ability), physical symptoms (e.g. fatigue, sleep disturbances, changes in appetite) as soon as suicidal ideations. Anxiety was assessed using the Generalized Anxiety Disorder questionnaire (GAD-7) [49], this 
Table 1

Clinical characteristics $(n=200)$.

\begin{tabular}{ll}
\hline & Mean (SD) \\
\hline Male/female & $67 / 133$ \\
Age & $18.72(1.85)$ \\
Clinical diagnosis & $167(83.5 \%)$ \\
Depressive disorder (F32.1, F32.2, F33.0, F33.1, F33.2) & $51(25.5 \%)$ \\
Anxiety disorder (F40.1, F41.0, F41.1, F41.2) & $26(13.0 \%)$ \\
Comorbidities & $124(66.5 \%)$ \\
PTSD & $19(9.5 \%)$ \\
Personality disorders (F60.30, F60.31, F60.4, F60.6, F60.7, F60.8, F61) & $33(16.5 \%)$ \\
Eating disorder (F50.0, F50.1, F50.2) & \\
Others (F42.1, F42.2, F45.1, F44.5, F90.0) & \\
\hline
\end{tabular}

Measures: The Structured Clinical Interview for the Diagnostic and statistical manual of mental disorders, 4th edition (DSM-IV) (SCID-I) and the Structured Clinical Interview for DSM-IV Axis II Personality Disorders II (SCID-II) were used to identify the clinical diagnoses.

7-item self-reported questionnaire can be used to detect individuals with generalized anxiety disorders and to estimate the severity of anxiety overall. On both scales, patients were asked to rate their symptoms for the last two weeks on a 4 point scale (0 - Not at all; 1 - Several days; 2 - More than half the days; 3 - Nearly every day), which can be calculated to a total sum within a range from 0 to 27 for depressive symptoms and 0 to 21 for anxiety. Cronbach's alpha for GAD-7 is 0.83 and for PHQ-9 - 0.80.

\subsection{Analysis}

Our main aim was to investigate the association between childhood trauma and PLEs and its mechanisms by considering BPD features, depression and anxiety as mediators of the relationship. The statistical analysis was conducted using Statistical Package for Social Sciences (SPSS) for Windows version 22 (IBM Statistics) and the Process macro [50] for SPSS. To investigate the relations between PLEs, childhood trauma and BPD features, as well anxiety and depression (psychopathologic variables) we first calculated Pearson's correlational coefficients. In addition, we performed multiple linear regression analysis to find out which variables of the ACE specifically predict the total number of PLEs (PQ-16 total score). To find out conditions that mediate the prevalence of PLEs in our subgroup, we carried out multiple regression analyses to test for associations between the independent variable (childhood trauma), mediators (BPD features, depression and anxiety) and the dependent variable (PLEs). Regarding the assumptions of multiple regressions analysis distributions of residuals were normal at every value of the dependent variables. Based on the tolerance value and the variance inflation factor (VIF) there were no problems with multicollinearity. Mediation analyses were conducted separately for BPD features, anxiety and depression using Model 4 form the Process tool [50]. To test whether the indirect effect of the independent variable on the outcome through the mediator is significant, we used bias-corrected bootstrap confidence intervals as suggested by Preacher and Hayes [51]. The Process macro generated 5000 bootstrap samples to obtain $95 \%$ bias-corrected bootstrap confidence intervals for the indirect effects. The indirect effect will be significant if the $95 \%$ confidence interval does not contain zero. To confirm the results we used the Sobel test as well [52,53]. Due to the low number of men in our subgroup we controlled for gender in all analysis.

\section{Results}

\subsection{Sample characteristics}

Table 1 presents the clinical characteristics of the sample. Men were underrepresented in this study (33.5\%). The majority of the sample has 2 or more Axis-I diagnoses (79.5\%) and $124(66.5 \%)$ of the cohort fulfilled criteria for a personality disorder; 25 (12.5\%) of the sample abuse cannabis, 16 (8.0\%) were addicted to it. Alcohol abuse was shown by 15 (7.5\%), and 13 (6.5\%) consumed multiple substances.

\subsection{Prevalence of PLEs and childhood trauma}

Table 2 presents an overview of the psychopathological characteristics of the sample. Fifty-four percent of the sample $(n=108)$ reached the cutoff of 6 endorsed items in the PQ-16; on average the score amounted to $6.43(S D=3.61)$. The authors [42] suggest to perform standardized prodromal testing when six or more items are endorsed. Most reported items were item 1 - "I feel uninterested in things I used to enjoy" ( $n=159,79.5 \%)$, Item 2 - "I often seem to live through events exactly as they happened before (de ja'-vu) ( $n=113,56.5 \%)$, item 5 - "I have been confused at times whether something I experiences was real or imaginary" $(n=120$, $60 \%$ ), item 7 - "I get extremely anxious when meeting people for the first time" ( $n=140,70 \%)$, Item 9 - "My thoughts are sometimes so strong that I can almost hear them" ( $n=100,50 \%)$, item 11 - "Sometimes I felt that I'm not in control of my own ideas or thoughts" $(n=126,63 \%)$, item 14 - "I often feel that others have it in for me" ( $n=111,55.5 \%)$.

Regarding childhood trauma patients reported on average exposure to $3.02(S D=2.40)$ traumatic events in their childhood. Most reported experiences were emotional neglect (50.5\%, $n=101)$, emotional abuse $(50 \%, n=100)$, grown up with a family member with mental illness $(47.5 \%, n=95)$, separation from a parent $(41.0 \%, n=82)$, physical abuse $(29.0 \%, n=58)$, substance abuse or dependence of a member of the household $(25.5 \%, n=51)$ and sexual abuse $(23.5 \%, n=47)$ in childhood (Table 3$)$.

\subsection{Correlational analysis}

To find out correlations between specific traumatic experiences measured by the ACE and PLEs we first performed a correlational analysis between all ACE items with the PQ-16 total score. Our analysis considered data from the total sample due to the low number of men in this study. For an overview of correlational coefficients see Fig. 1.

Table 2

Psychopathological characteristics $(n=200)$.

\begin{tabular}{ll}
\hline & $M(S D)$ \\
\hline PLEs & $6.43 \pm 3.61$ \\
ACE & $3.02 \pm 2.40$ \\
BSL-23 & $40.21 \pm 21.28$ \\
PHQ-9 & $16.23 \pm 5.41$ \\
GAD-7 & $12.15 \pm 4.80$ \\
\hline
\end{tabular}


Table 3

Correlational analysis $(n=200)$.

\begin{tabular}{|c|c|c|c|c|c|c|c|}
\hline & $M$ & $S D$ & PQ-16 total & ACE total & GAD-7 total & BSL-23 total & PHQ-9 total \\
\hline PHQ-9 & 16.33 & 5.43 & $0.48\left({ }^{* *}\right)$ & $0.27\left({ }^{* *}\right)$ & $0.72(* *)$ & $0.74\left({ }^{* *}\right)$ & - \\
\hline BSL-23 & 40.59 & 21.47 & $0.56\left(^{* *}\right)$ & $0.42(* *)$ & $\left.0.699^{* *}\right)$ & - & $0.74(* *)$ \\
\hline GAD-7 & 12.22 & 4.88 & $0.42\left(^{* *}\right)$ & $0.24\left({ }^{* *}\right)$ & - & $0.69\left({ }^{* *}\right)$ & $0.72(* *)$ \\
\hline ACE total & 3.04 & 2.37 & $0.33(* *)$ & - & $0.24\left({ }^{* *}\right)$ & $0.42(* *)$ & $0.27(* *)$ \\
\hline PQ-16 total & 6.43 & 3.68 & - & $0.33(* *)$ & $0.42\left({ }^{* *}\right)$ & $0.56\left({ }^{* *}\right)$ & $0.48\left({ }^{* *}\right)$ \\
\hline
\end{tabular}

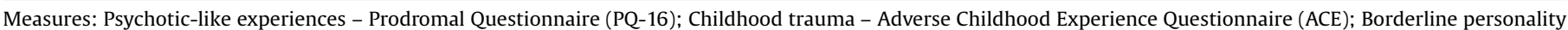

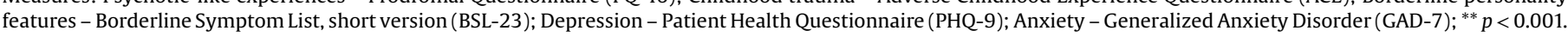

Further, we performed correlational analyses for the total sample to examine the connections between symptoms of depression and anxiety, borderline personality features, childhood trauma and PLEs (see Fig. 1). All three symptom dimensions as well as childhood trauma were associated significantly with PQ-16 total, but the association with Borderline personality features (BSL-23) was strongest $(r=0.56, p<0.001)$.

\subsection{Mediation}

To find out which variables mediate the relation between PLEs and childhood trauma we initially performed mediation analysis using Hayes'Process macro in SPSS with one mediator (Model 4). The results confirmed a significant association between childhood trauma with PLEs $(\beta=.30, p<0.001)$, which was attenuated and changed into a non-significant association when taking into account the mediator borderline personality features $(B=0.12, n . s)$. A bootstrapped confidence interval $(\mathrm{CI})$ for the indirect effect $(\mathrm{ACE} \rightarrow \mathrm{BSL}-23 \rightarrow \mathrm{PQ}-16)$ based on 5000 bootstrap samples did not contain zero, thus indicating the significance of the indirect effect (CI $[0.17,0.43])$. The result indicates a full mediation effect, because after including borderline personality features in the model, the association between childhood trauma and PLE's became insignificant. The Sobel test also confirmed that the indirect path was significant $(Z=4.47, p<0.001)$.

Additionally, we repeated the mediation analysis and operationalized Borderline personality features by the SCID-II-Screening score to confirm the result that Borderline personality features are mediating the relationship between childhood trauma and PLEs. The analysis again demonstrated a full mediation. The inclusion of the mediator into the model attenuated the effect to non-significance $(~ B=0.15, n . s)$. A bootstrapped confidence interval $(\mathrm{CI})$ for the indirect effect $(\mathrm{ACE} \rightarrow \mathrm{SCID}-\mathrm{II} \rightarrow \mathrm{PQ}-16$ ) based on 5000 bootstrap samples did not contain zero, thus indicating the significance of the indirect effect (CI $[0.18,0.43])$. Sobel test confirmed the result $(Z=2.06, p<0.05)$.

Furthermore, the inclusion of depression in the model showed a partial effect. Depression attenuates the association between childhood trauma and PLEs significantly $(ß=0.22, p<0.001$;
CI $[0.07,0.26] ; Z=3.00 ; p<0.01)$. Equally, Anxiety could show a partial mediation after inclusion in the model $(\beta=0.22, p<0.001$, CI $[0.05,0.23] ; Z=2.74 ; p<0.01)$. Both results are indicating a significant reduction of the effect of childhood trauma on PLE's. Fig. 2 shows an overview of the three separate mediation analysis we performed to examine the influence of each mediator on the relation between childhood trauma and PLE's.

\section{Discussion}

The present study aimed at investigating the relationship between PLEs and psychopathological features and childhood trauma among non-psychotic adolescent help-seekers. In particular, we were interested in the mechanisms of the well-established relationship between exposure to childhood trauma and PLEs among non-psychotic adolescents seeking for psychiatric care. Based on the existing literature [39,54-56] we hypothesized that borderline personality features may mediate the relationship. In addition, we considered also mediating role of other psychopathological symptoms - depression and anxiety. To our knowledge, this is the first study examining the mechanisms of the relation of self-reported PLEs and childhood trauma with reference to the mediating role of borderline personality features, depression, and anxiety in a clinical sample of adolescents with non-psychotic psychiatric disorders.

In line with previous studies [11,14], our findings showed that self-reported PLEs are highly prevalent among adolescents with non-psychotic psychiatric disorders treated in inpatient psychiatric setting. More than half of the sample (54\%) reported six or more positive PLEs and reached the cutoff for performing further examinations of the risk states of psychosis as recommended by the authors [42]. At the same time, PLEs were significantly related to exposures to childhood trauma, which corroborate prior studies [32,34-36]. We observed that emotional neglect and sexual abuse were particularly associated with PLEs suggesting that the type of trauma could be relevant for the development of psychotic-like experiences [39,57-59].

Despite the convincing evidence showing a relationship between childhood adversities and PLEs [32-36], the mechanisms

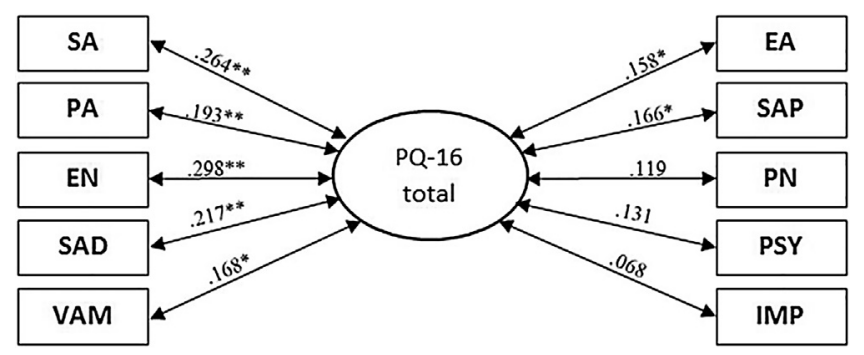

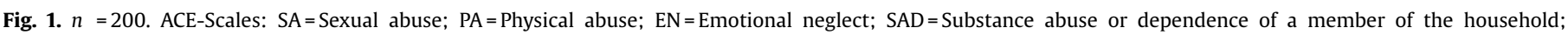

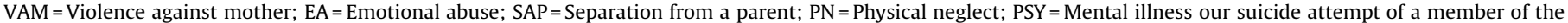
household; IMP = Imprisonment of a member of the household.

** $p<$. 0.001. * $p<0.01$. 


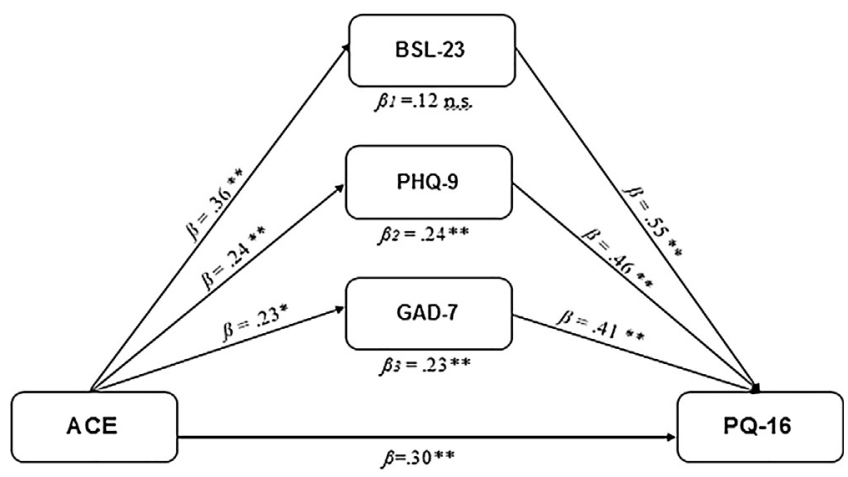

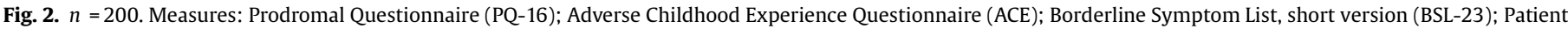

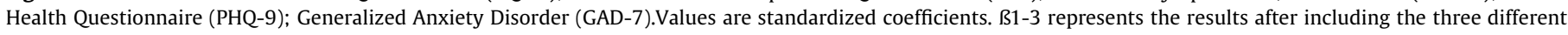
mediators. All variables were controlled for gender.

${ }^{* *} p<0.001 * p<0.01$.

for this remain poorly understood. In particular, there is still a limited number of studies investigating the mechanisms of the relationship among adolescent help-seekers. Our study, for the very first time, showed that borderline personality features fully mediate the relationship between childhood trauma and PLEs among non-psychotic adolescent help-seekers. At the same time, other psychopathological domains, depression and anxiety, mediated the relationship only partially. Hence, our findings confirm the significant role of dysfunctional personality features, in particular, borderline traits, in the development of PLEs [60,61]. Hence, in the context of mechanisms of the relationship between childhood trauma and PLEs, our results suggest borderline personality features as an important factor. For being more specific in the examination of borderline personality features and to include subthreshold features of a BPD without fulfilling entire diagnostic categories we decided to use the BSL-23 questionnaire. To confirm the effect of the mediation analysis we repeated the analysis with borderline personality features operationalized by SCID-II Screening questionnaire which showed full mediation effect equally (see supplement).

Our results may be interpreted in the way that development of a borderline personality constitution resulting from exposure to childhood trauma $[25,37,38]$ may contribute to a higher risk of PLEs under stress $[62,63]$. Previous results indicated that patients with BPD often suffer from multiple trauma $[64,65]$ and that in particular cumulative exposure to traumatic experiences were associated with significantly higher levels of PLEs in BPD $[20,66]$ and in general population [59,67-70]. Exposure to multiple early-life trauma could lead to a sensitization in sense of a higher physiological sensitivity, based on neurodevelopmental changes like a dysfunction within the midbrain dopaminergic system [71,72] which could lead to an increased sensitivity to minor stresses in daily life and a higher susceptibility to subtle psychotic-like reactions in response to stress $[73,74]$. Thus, it could be assumed that neurodevelopmental sensitization associated with childhood trauma can make individuals with BPD or borderline personality features more prone for psychotic-like reactions especially in consequence of stress $[20,66,74]$. Previous studies confirmed that patients with BPD presented the strongest psychotic reactivity to daily life stress in comparison to patients with cluster $C$ personality disorder, patients with psychotic disorder or healthy controls and particularly interpersonal stress increase the prevalence of PLEs in patients with BPD [25,62]. Further, longitudinal studies with multiple assessment points will likely foster our understanding of the interplay between childhood trauma, borderline personality features, and PLEs.

As mentioned above, depression and anxiety mediated the relationship between childhood trauma and PLEs partially. Some studies emphasized the particular role of anxiety and depression regarding the relationship of traumatic experiences and PLEs [35,39]. Moreover, our sample was heterogeneous regarding the diagnosis therefore we verified the influence of these symptom dimensions as well. Furthermore, the scale we used to assess borderline personality features was very specific and does not cover for anxiety and depression. The results could be interpreted against the backdrop of childhood trauma. It could be assumed that the emerging of depression and/or anxiety can influence the development of PLEs independently from a borderline personality constitution. Further studies should examine the role of anxiety and depression regarding childhood trauma and PLEs in adolescent patients.

The results should be interpreted in light of some limitations. First, the cross-sectional design of our study precludes firm causal inferences. Further longitudinal studies are required to establish causal relationships. It should be however noted that the causality in psychiatry is often circular [75]. Second, the prevalence of PLEs was based on a self-report questionnaire and not verified by a clinical interview. Studies suggesting that the measurement of PLEs in community samples using self-report scales generally showed higher rates than in interview-based studies, however, there are no differences regarding validity or reliability [76], selfreport instrument rather accurately predict interview recorded psychotic experiences [1]. However, the absence of a clinical interview to validate the PQ-16 can lead to some misinterpretations of the variables because some of them could be interpreted in the light of patient's current symptoms then to PLEs which could falsify the total score. For example item 7 -"I get extremely anxious when meeting people for the first time" would also be affirmed by patients with social phobia. This clarified the necessity to the rather use of interview based studies.

Third, we did not perform further investigations if patients reached the cutoff ( $\geq 6$ endorsed items) [42] to verify a current ultra-high-risk for psychosis (UHR).

The previous study showed that borderline personality features did not increase the risk of psychosis among those patients who fulfilled criteria for UHR state [60]. Our results rather suggested that the prevalence of borderline personality features, even if they are sub-clinical could indicate different origin and course of UHR symptoms and should be taking into account in patients with UHR to improve diagnostic accuracy and prognostic estimation. Equally symptoms of depression and anxiety shall be valued in the evaluation of ultra-high-risk states. Furthermore, men were distinctly underrepresented in our study. This is reflected in a German health monitoring of public attitudes toward psychiatric and psychotherapeutic treatment which shows a lesser claim by men than by women [77]. 
Finally, we did not capture dissociative symptoms separately, and they may confound with PLEs. We were particularly interested in the role of borderline personality features in the relationship between childhood trauma and PLEs, and thus we did not include some of the important factors that have been shown to play a role in previous studies $[55,78,79]$. For instance, recent studies suggest that cognitive biases may also be an important factor that mediates the relationship between exposures to trauma and PLEs [31,78]. Of importance, cognitive biases related to psychosis have been observed also among patients with BPD [21]. Following investigations would benefit from considering these factors and limitations of our study.

To conclude, our study suggests borderline personality features as an important factor in the relationship between childhood trauma and PLEs among non-psychotic adolescent help-seekers. These findings may have important theoretical and clinical implications. For instance, considering the interplay between borderline personality features and exposure to traumatic events among those who report PLEs may have a potential to increase sensitivity of prediction of the risk states for developing psychosis, as well as lower social functioning or higher risk of suicidality and non-suicidal self-injury $[27,80]$. However, further investigations are warranted.

\section{References}

[1] Kelleher I, Cannon M. Psychotic-like experiences in the general population: characterizing a high-risk group for psychosis. Psychol Med 2011;41:1-6.

[2] Van Os J, Hanssen M, Bijl RV, Strauss Ravelli A. revisited: a psychosis continuum in the general population? Schizophr Res 1969;45 (1)11-20 2000

[3] Kelleher I, Connor D, Clarke MC, Devlin N, Harley M, Cannon M. Prevalence of psychotic symptoms in childhood and adolescence: a systematic review and meta-analysis of population-based studies. Psychol Med 2012;42(9):1857-63.

[4] Yung AR, Nelson B, Baker K, Buckby JA, Baksheev G, Cosgrave EM. Psychoticlike experiences in a community sample of adolescents: implications for the continuum model of psychosis and prediction of schizophrenia. Aust N Z J Psychiatry 2009;2:118-28.

[5] Schultze-Lutter F, Michel C, Ruhrmann S, Schimmelmann BG. Prevalence and clinical significance of DSM-5-attenuated psychosis syndrome in adolescents and young adults in the general population: the Bern Epidemiological At-Risk (BEAR) study. Schizophr Bull 2014;6:1499-508.

[6] van Os J, Linscott RJ, Myin-Germeys I, Delespaul P, Krabbendam L. A systematic review and meta-analysis of the psychosis continuum: evidence for a psychosis proneness-persistence-impairment model of psychotic disorder. Psychol Med 2009;39:179-95.

[7] Werbeloff N, Drukker M, Dohrenwend BP, Levav I, Yoffe R, van Os J, et al. Selfreported attenuated psychotic symptoms as forerunners of severe mental disorders later in life. Arch Gen Psychiatry 2012;5:467-75.

[8] Yung AR, Buckby JA, Cotton SM, Cosgrave EM, Killackey EJ, Stanford C, et al Psychotic-like experiences in nonpsychotic help-seekers: associations with distress, depression, and disability. Schizophr Bull 2006;32:352-9.

[9] Armando M, Nelson B, Yung AR, Ross M, Birchwood M, Girardi P, et al. Psychotic-like experiences and correlation with distress and depressive symptoms in a community sample of adolescents and young adults. Schizophr Res 2010;119:258-65.

[10] Armando M, Saba R, Monducci E, Papaleo F, Dario C, Righetti V, et al. Subtypes of psychotic-like experiences in a community sample of young adults: sociodemographic correlates and substance use. Riv Psichiatr 2012;47:424-31.

[11] Kelleher I, Devlin N, Wigman JT, Kehoe A, Murtagh A, Fitzpatrick C, et al. Psychotic experiences in a mental health clinic sample: implications for suicidality, multimorbidity and functioning. Psychol Med 2014;44 (8):1615-24.

[12] Kelleher I, Keeley H, Corcoran P, Lynch F, Fitzpatrick C, Devlin N, et al Clinicopathological significance of psychotic experiences in non-psychotic young people: evidence from four population-based studies. Br J Psychiatry 2012;201(1):26-32.

[13] Wigman JT, van Nierop M, Vollebergh WA, Lieb R, Beesdo-Baum K, Wittchen $\mathrm{HU}$, et al. Evidence that psychotic symptoms are prevalent in disorders of anxiety and depression, impacting on illness onset, risk, and severity implications for diagnosis and ultra-high risk research. Schizophr Bull 2012;38:247-57.

[14] Pontillo M, De Luca M, Pucciarini ML, Vicari S, Armando M. All that glitters is not gold: prevalence and relevance of psychotic-like experiences in clinical sample of children and adolescents aged 8-17 years old. Early Interv Psychiatry 2016;12(4):702-7.

[15] Brandizzi M, Schultze-Lutter F, Masillo A, Lanna A, Curto M, Lindau JF, et al. Self-reported attenuated psychotic-like experiences in help-seeking adolescents and their association with age, functioning and psychopathology. Schizophr Res 2014;160(1):110-7.

[16] Hodgekins J, Lower R, Wilson J, Cole H, Ugochukwu U, Maxwell S, et al. Clinician-rated and self-reported psychotic-like experiences in individuals accessing a specialist Youth Mental Health Service. Br J Clin Psychol 2018;57 (3):367-81.

[17] Varghese D, Scott J, Welham J, Bor W, Najman J, O'Callaghan M, et al. Psychoticlike experiences in major depression and anxiety disorders: a populationbased survey in young adults. Schizophr Bull 2011;37(2):389-93.

[18] Nam B, Hilimire M, Schiffman J, DeVylder J. Psychotic experiences in the context of depression: the cumulative role of victimization. J Psychiatr Res 2016;82:136-40.

[19] Barragan M, Laurens KR, Navarro JB, Obiols JE. Psychotic-like experiences and depressive symptoms in a community sample of adolescents. Eur Psychiatry 2011;26(6):396-401.

[20] Barnow S, Arens EA, Sieswerda S, Dinu-Biringer R, Spitzer C, Lang S. Borderline personality disorder and psychosis: a review. Curr Psychiatry Rep 2010;12 (3):186-95.

[21] Moritz S, Schilling L, Wingenfeld K, Köther U, Wittekind C, Terfehr K, et al. Psychotic-like cognitive biases in borderline personality disorder. J Behav Ther Exp Psychiatry 2011;42(3):349-54.

[22] Merrett Z, Rossell SL, Castle DJ. Comparing the experience of voices in borderline personality disorder with the experience of voices in a psychotic disorder: a systematic review. Aust N Z J Psychiatry 2016;50(7):640-8.

[23] Niemantsverdriet MB, Slotema CW, Blom JD, Franken IH, Hoek HW, Sommer IE, et al. Hallucinations in borderline personality disorder: prevalence, characteristics and associations with comorbid symptoms and disorders. Sci Rep 2017;7(1):13920.

[24] Pearse LJ, Dibben C, Ziauddeen H, Denman C, McKenna PJ. A study of psychotic symptoms in borderline personality disorder. J Nerv Ment Dis 2014;202 (5):368-71.

[25] Schroeder K, Fisher HL, Schäfer I. Psychotic Symptoms in patients with borderline personality disorder: prevalence and clinical management. Curr Opin Psychiatry 2013;26:113-9.

[26] Zonnenberg C, Niemantsverdriet MB, Blom JD, Slotema CW. Auditory verbal hallucinations in patients with borderline personality disorder. Tijdschr Psychiatr 2015;58:122-9.

[27] Schroeder K, Schätzle A, Kowohl P, Leske L, Huber CG, Schäfer I. [Prevalence and phenomenology of psychotic-like symptoms in borderline personality disorder - association with suicide attempt and use of psychiatric inpatient treatement]. Psychother Psychosom Med Psychol 2018;68:516-42.

[28] Stern AJ. Psychoanalytic investigation of and therapy in the borderline group of neuroses. Psychoanal Q 1938;7(4):467-89.

[29] Gunderson JG, Singer MT. Defining borderline patients: an overview. Am J Psychiatry 1975;132:1-10.

[30] Masterson JF, Rinsley DB. The borderline syndrome: the role of the mother in the genesis and psychic structure of the borderline personality. Int J Psychoanal 1975;56(2):163-77.

[31] Gawęda Ł, Prochwicz K, Adamczyk P, Frydecka D, Misiak B, Kotowicz K, et al. The role of self-disturbances and cognitive biases in the relationship between traumatic life events and psychosis proneness in a non-clinical sample. Schizophr Res 2018;193:218-24.

[32] Gibson LE, Alloy LB, Ellman LM. Trauma and the psychosis spectrum: a review of symptom specificity and explanatory mechanisms. Clin Psychol Rev 2016;49:92-105.

[33] Skehan D, Larkin W, Read J. Childhood adversity and psychosis: a literature review with clinical and societal implications. Psychoanal Cult Soc 2012;17 (4):373-91.

[34] Kelleher I, Harley M, Lynch F, Arseneault L, Fitzpatrick C, Cannon M. Associations between childhood trauma, bullying and psychotic symptoms among a school-based adolescent sample. Br J Psychiatry 2008;193(5):37882.

[35] Mackie CJ, Castellanos-Ryan N, Conrod PJ. Developmental trajectories of psychotic-like experiences across adolescence: impact of victimization and substance use. Psychol Med 2011;41(1):47-58.

[36] Cristóbal-Narváez P, Sheinbaum T, Ballespí S, Mitjavila M, Myin-Germeys I, Kwapil TR. Impact of adverse childhood experiences on psychotic-like symptoms and stress reactivity in daily life in nonclinical young adults. PloS One 2016;11(4)e0153557.

[37] Ball JS, Links PS. Borderline personality disorder and childhood trauma: evidence for a causal relationship. Curr Psychiatry Rep 2009;11(1):63-8.

[38] Adams B, Sanders T. Experiences of psychosis in borderline personality disorder: a qualitative analysis. J Ment Health 2011;20(4):381-91.

[39] Freeman D, Fowler D. Routes to psychotic symptoms: trauma, anxiety and psychosis-like experiences. Psychiatry Res 2009;169(2):107-12.

[40] Wittchen HU, Zaudig M, Skid Fydrich T. Strukturiertes klinisches interview für DSM-IV. Achse I und II. Handanweisung. Göttingen. Hogrefe; 1997.

[41] Fydrich T, Renneberg B, Schmitz B, HU. SKID Wittchen II. Strukturiertes Klinisches Interview für DSM-IV, Achse II: Persönlichkeitsstörungen. Interviewheft. Eine deutschsprachige, erw. Bearb. d. amerikanischen Originalversion d. SKID-II von: MB First, RL Spitzer, M. Gibbon, JBW Williams, L. Benjamin (Version 3/96). Göttingen: Hogrefe 1997.

[42] Ising HK, Veling W, Loewy RL, Rietveld MW, Rietdijk J, Dragt S, et al. The validity of the 16-item version of the Prodromal Questionnaire (PQ-16) to screen for ultra high risk of developing psychosis in the general help-seeking population. Schizophr Bull 2012;38(6):1288-96. 
[43] Loewy RL, Johnson JK, Cannon TD. Self-report of attenuated psychotic experiences in a college population. Schizophr Res 2007;93(1):144-51.

[44] Schäfer I, Wingenfeld K, ACE-D Spitzer C. Deutsche version des Adverse childhood experiences questionnaire. In: Richter D, Brähler E, Strauß B, editors. Diagnostische verfahren in der sexualwissenschaft. Göttingen: Hogrefe; 2014. p. 11-5.

[45] Bohus M, Kleindienst N, Limberger MF, Stieglitz RD, Domsalla M, Chapman AL, et al. The short version of the Borderline Symptom List (BSL-23): development and initial data on psychometric properties. Psychopathology 2009;42(1):32-9.

[46] Bohus M, Limberger MF, Frank U, Chapman AL, Kühler T, Stieglitz RD. Psychometric properties of the borderline symptom list (BSL). Psychopathology 2007;40(2):126-32.

[47] Gräfe K, Zipfle S, Herzog W, Löwe B. Screening psychischer Störungen mit dem Gesundheitsfragebogen für Patienten (PHQ-D). Diagnostica 2004;50 (4):171-81.

[48] Kroenke K, Spitzer RL, Williams JB. The PHQ-9. J Gen Intern Med 2001;16 (9):606-13.

[49] Spitzer RL, Kroenke K, Williams JB, Löwe B. A brief measure for assessing generalized anxiety disorder: the GAD-7. Arch Intern Med 2006;166 (10):1092-7.

[50] Hayes AF. Introduction to mediation, moderation, and conditional process analysis: a regression-based approach. New York: Guilford Press; 2013.

[51] Preacher KJ, Hayes AF. Asymptotic and resampling strategies for assessing and comparing indirect effects in multiple mediator models. Behav Res Methods Instrum Comput 2008;40(3):879-91.

[52] Preacher KJ, Hayes AF. SPSS and SAS procedures for estimating indirect effects in simple mediation models. Behav Res Methods Instrum Comput 2004;36 (4):717-31.

[53] Sobel ME. Asymptotic confidence intervals for indirect effects in structural equation models. Sociol Methodol 1982;13:290-312.

[54] Baryshnikov I, Aaltonen K, Suvisaari J, Koivisto M, Heikkinen M, Joffe G, et al Features of borderline personality disorder as a mediator of the relation between childhood traumatic experiences and psychosis-like experiences in patients with mood disorder. Eur Psychiatry 2018;49:9-15.

[55] Fisher HL, Schreier A, Zammit S, Maughan B, Munafò MR, Lewis G, et al. Pathways between childhood victimization and psychosis-like symptoms in the ALSPAC birth cohort. Schizophr Bull 2012;39(5):1045-55.

[56] Fisher HL, Appiah-Kusi E, Grant C. Anxiety and negative self-schemas mediate the association between childhood maltreatment and paranoia. Psychiatry Res 2012;196(2):323-4.

[57] Bebbington P, Jonas S, Kuipers E, King M, Cooper C, Brugha T, et al. Childhood sexual abuse and psychosis: data from a cross-sectional national psychiatric survey in England. Br J Psy 2011;199(1):29-37.

[58] Arseneault L, Cannon M, Fisher HL, Polanczyk G, Moffitt TE, Caspi A. Childhood trauma and children's emerging psychotic symptoms: a genetically sensitive longitudinal cohort study. Am J Psychiatry 2011;168(1):65-72.

[59] McGrath JJ, McLaughlin KA, Saha S, Aguilar-Gaxiola S, Al-Hamzawi A, Alonso J, et al. The association between childhood adversities and subsequent first onset of psychotic experiences: a cross-national analysis of 23998 respondents from 17 countries. Psychol Med 2017;47(7):1230-45.

[60] Ryan J, Graham A, Nelson B, Yung A. Borderline personality pathology in young people at ultra high risk of developing a psychotic disorder. Early Interv Psychiatry 2017;11(3):208-14.

[61] Baryshnikov I, Suvisaari J, Aaltonen K, Koivisto M, Melartin T, Näätänen P, et al. Self-reported psychosis-like experiences in patients with mood disorders. Eur Psychiatry 2018.
[62] Glaser JP, Van Os J, Thewissen V, Myin-Germeys I. Psychotic reactivity in borderline personality disorder. Acta Psychiatr Scand 2010;121(2):125-34.

[63] Oliva F, Dalmotto M, Pirfo E, Furlan PM, Picci RL. A comparison of thought and perception disorders in borderline personality disorder and schizophrenia: psychotic experiences as a reaction to impaired social functioning. BMC Psychiatry 2014;14:239.

[64] Temes CM, Magni LR, Fitzmaurice GM, Aguirre BA, Goodman M, Zanarini MC. Prevalence and severity of childhood adversity in adolescents with BPD, psychiatrically healthy adolescents, and adults with BPD. Personal Ment Health 2017;11(3):171-8.

[65] Pietrek C, Elbert T, Weierstall R, Müller O, Rockstroh B. Childhood adversities in relation to psychiatric disorders. Psychiatry Res 2013;206(1):103-10.

[66] Gras A, Amad A, Thomas P, Jardri R. Hallucinations and borderline personality disorder: a review. Encephale 2014;40(6):431-8.

[67] Janssen I, Krabbendam L, Bak M, Hanssen M, Vollebergh W, Graaf RD, et al. Childhood abuse as a risk factor for psychotic experiences. Acta Psychiatr Scand 2004;109(1):38-45.

[68] Spauwen J, Krabbendam L, Lieb R, Wittchen HU, Van Os J. Impact of psychological trauma on the development of psychotic symptoms: relationship with psychosis proneness. Br J Psychiatry 2006;188(6):527-33.

[69] Whitfield CL, Dube SR, Felitti VJ, Anda RF. Adverse childhood experiences and hallucinations. Child Abuse Negl 2005;29(7):797-810.

[70] Shevlin M, Murphy J, Read J, Mallett J, Adamson G, Houston JE. Childhood adversity and hallucinations: a community-based study using the National Comorbidity Survey Replication. Soc Psychiatry Psychiatr Epidemiol 2011;46 (12):1203-10.

[71] Howes OD, Murray RM. Schizophrenia: an integrated sociodevelopmentalcognitive model. Lancet 2014;383(9929):1677-87.

[72] Nicol K, Pope M, Romaniuk L, Hall J. Childhood trauma, midbrain activation and psychotic symptoms in borderline personality disorder. Transl Psychiatry 2015;5(5):e559.

[73] Myin-Germeys I, van Os J. Stress-reactivity in psychosis: evidence for an affective pathway to psychosis. Clin Psychol Rev 2007;27(4):409-24.

[74] Glaser JP, Van Os J, Portegijs PJ, Myin-Germeys I. Childhood trauma and emotional reactivity to daily life stress in adult frequent attenders of general practitioners. J Psychosom Res 2006;61(2):229-36.

[75] Young G. Causality in psychiatry: a hybrid symptom network construct model. Front Psychiatry 2015;6:164.

[76] Linscott RJ, Van Os J. An updated and conservative systematic review and meta-analysis of epidemiological evidence on psychotic experiences in children and adults: on the pathway from proneness to persistence to dimensional expression across mental disorders. Psychol Med 2013;43 (6):1133-49.

[77] Rommel A, Bretschneider J, Kroll LE, Prütz F, Thom J. Inanspruchnahme psychiatrischer und psychotherapeutischer Leistungen - individuelle Determinanten und regionale Unterschiede. J Health Monitor 2017;2:4

[78] Gibson LE, Reeves LE, Cooper S, Olino TM, Ellman LM. Traumatic life event exposure and psychotic-like experiences: A multiple mediation model of cognitive-based mechanisms. Schizophr Res 2018, doi:http://dx.doi.org/ 10.1016/j.schres.2018.02.005.

[79] Gibson LE, Anglin DM, Klugman JT, Reeves LE, Fineberg AM, Maxwell SD, et al. Stress sensitivity mediates the relationship between traumatic life events and attenuated positive psychotic symptoms differentially by gender in a college population sample. J Psychiatr Res 2014;53:111-8.

[80] Koyanagi A, Stickley A, Haro JM. Subclinical psychosis and suicidal behavior in England: findings from the 2007 adult psychiatric morbidity survey. Schizophr Res 2015;168(1):62-7. 